\title{
GAMMA-RAY BURSTS AND THEIR CENTRAL ENGINES
}

\author{
Stephan Rosswog \\ School of Engineering and Science \\ International University Bremen \\ Germany \\ s.rosswog@iu-bremen.de
}

\begin{abstract}
Gamma-ray bursts are the most luminous and probably the most relativistic events in the universe. The last few years have seen a tremendous increase in our knowledge of these events, but the source of the bursts still remains elusive. I will summarise recent progress in this field with special emphasis on our understanding of the possible progenitor systems.
\end{abstract}

Keywords: gamma rays: bursts; supernovae; stars: neutron; radiation processes: non-thermal; dense matter; hydrodynamics; neutrinos; magnetic fields

\section{Introduction}

Like some other spectacular discoveries such as the cosmic microwave background, gamma-ray bursts (GRBs) were discovered by accident. Meant to monitor the "outer space treaty", the American VELA satellites detected in July 1967 an intense flash of gamma-rays of unknown origin. It took until 1973 before the first detected GRBs were published for the scientific community (Klebesadel et al. 1973).

This caused furious research activity and a flurry of often very exotic theoretical models. At the Texas conference in 1974, only one year after the first scientific publication, Malvin Ruderman summarised the situation: "The only feature that all but one (and perhaps all) of the very many proposed models have in common is that they will not be the explanation of GRBs. Unfortunately, limitations of time prevent me from telling you which model is the exception....". But his favourite model, accretion onto a black hole, is still a promising horse in today's race.

\section{Observations}

GRBs are short flashes of gamma-rays that outshine for short moment the whole rest of the gamma-ray sky. The BATSE instruments on board the Comp- 


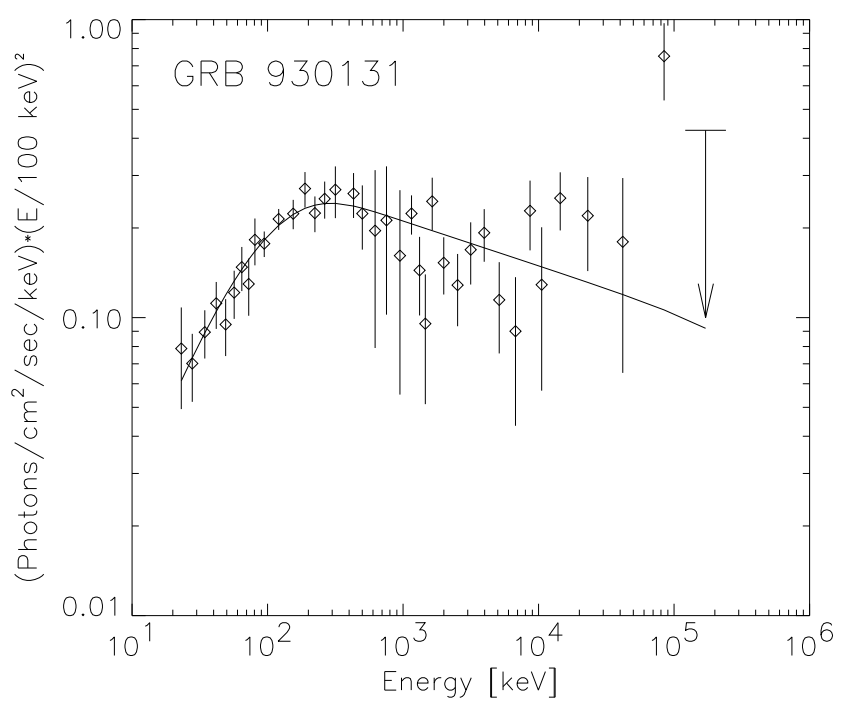

Figure 1. Spectrum of GRB930131 together with a synchrotron model fit (Bromm \& Schaefer 1999).

ton Gamma-Ray Observatory observed bursts at a rate of around one event per day (Paciesas et al. 1999). Opposite to initial expectations BATSE detected an inhomogeneous, but highly isotropic distribution of burst sources. GRB lightcurves exhibit a tremendous variety ranging from single featureless spikes, over "FREDS" (fast rise exponential decay) to completely erratic sequences of pulses. The lightcurves vary on millisecond time scales, the shortest variability time scale so far being $0.22 \mathrm{~ms}$ for GRB920229 (Schaefer and Walker 1999). The spectra of GRBs are non-thermal (see Figure 1) with high-energy tails extending up to several GeV. The spectra can be accurately fitted with an exponentially smoothed, broken powerlaw, the so-called "Band-function" (Band et al. 1993). The "knee" where both powerlaws are joined is referred to as the break energy. Typically break energies lie around several hundred keV. Generally, the lower spectral energy index is compatible with a synchrotron origin of the radiation (Cohen et al. 1997), there are however some bursts with a lower energy slope steeper than predicted by the synchrotron model (Preece et al. 1998). The duration distribution of GRBs is bimodal with a first peak at $\sim 0.2$ $\mathrm{s}$ and a second one at $\sim 30 \mathrm{~s}$. Bursts below (above) $2 \mathrm{~s}$ are referred to as "short (long) bursts" (Kouveliotou et al. 1993). Bursts of the short category exhibit predominately harder spectra than their long-duration cousins. The hardness is usually measured via the "hardness ratio", the ratio of photon counts in a high and a low energy channel of BATSE. 
The discovery of the first redshifts (Costa et al. 1997) in GRB afterglows settled a long debate about whether they occur at cosmological distances or not. By now, the cosmological origin of long GRBs is a well-established fact. Searches for the host galaxies found long bursts occurring predominantly in galaxies with active star formation (Bloom et al. 2002), pointing to a rather short-lived progenitor system, possibly related to the death of massive stars that die close to their birth-places. A GRB supernova connection had been suspected when in the localisation error box of the BeppoSAX wide field camera of GRB980425 a supernova of type Ib/c was found. By now, "bumps" have been detected in the optical afterglow lightcurves of several long GRBs (e.g. Bloom et al. 1999). These bumps were interpreted as the lightcurves from an underlying supernova that occurred roughly at the same time as the GRB. The idea of a long GRB supernova connection has convincingly been corroborated by the detection of a very energetic supernova that occurred temporally and spatially coincident with GRB030329 (Hjorth et al. 2003, Stanek et al. 2003). Thus (at least some of the) long bursts seem to go along directly with a corecollapse supernova.

Short GRBs are usually assumed to be cosmological as well, but we are currently still lacking direct evidence in the form of afterglow observations. There are indications that the observed short GRBs occurred possibly at somewhat shorter distances (Mao et al. 1994). This would be consistent with compact binary sources that merge relatively late in the age of the universe due to their long inspiral time (Fryer et al. 1999).

The fluences of typically $10^{-5}$ to $10^{-7} \mathrm{erg} / \mathrm{cm}^{2}$ and cosmological redshifts observed so far imply isotropic burst energies of up to $\sim 4 \cdot 10^{54} \mathrm{ergs}$ for GRB990123, corresponding to twice rest mass energy of the sun in gammarays alone. This "energy crisis" pointed to beamed emission. By now, in several GRBs achromatic breaks have been identified from which (under certain assumptions) jet opening angles of $\sim 5^{\circ}$ have been derived (Frail et al. 2001). Taking beaming angle corrections into account, the energy requirements are alleviated, the initially huge range of isotropic energies ranging from a few times $10^{51}$ to $4 \cdot 10^{54}$ ergs collapses to a narrow distribution around $\sim 5 \cdot 10^{50} \mathrm{erg}$. If true, this allows the use of GRBs as standard candles to infer, for example, star formation in the high redshift universe (e.g. Lloyd-Ronning et al. 2002). We briefly want to mention two exciting, but currently still controversial topics: X-ray emission lines and polarisation of the prompt GRB-emission. X-ray emission features have been reported for several X-ray afterglows (e.g. Piro et al. 2000). The confidence level for these detections, however, is rather low. The energy contained in these lines is very large, $\sim 10^{49} \mathrm{erg}$. Since the efficiency to produce an X-ray emission line cannot exceed $1 \%$ the burst would have to have $\sim 10^{51} \mathrm{ergs}$ in X-rays (Ghisellini et al. 2002), which sets a strict lower limit on the burst energy that is hard to reconcile with the results of Frail 
et al. (2001).

Using RHESSI results Coburn and Boggs (2003) reported on a high linear polarisation of the prompt emission of GRB021206, $\Pi=0.8 \pm 0.2$. This could point to the interesting possibility of magnetic fields being advected from the central source rather than being generated in shocks. A re-analysis of the same data, however, found a no clear indication of for polarisation of GRB021206 (Rutledge and Fox, 2003).

\section{The fireball model}

The above listed observational facts impose constraints on the progenitor system(s) responsible for gamma-ray bursts.

The first one, often referred to as the "compactness-problem" comes from the non-thermal GRB spectra and was originally used as an argument against a possible cosmological origin of GRBs (Ruderman 1975, Schmidt 1978). The millisecond variability of the lightcurves points to a compact source for the GRB, as from simple causality arguments the dimension of the system should be $D<c \cdot \delta t \approx 300 \mathrm{~km}$. But if an energy of $\sim 10^{51} \mathrm{erg}$ in $\sim 1 \mathrm{MeV}$ photons is released in such a small volume the optical depth to pair creation must be huge: $\tau \sim 10^{13}$ and therefore one would expect a thermal spectrum, in blatant contrast to the observations. The way out of this dilemma is relativistic motion (Paczynski 1986, Goodman 1986). If the emission is coming from material moving with Lorentz factor $\Gamma$ towards the observer, the source can be larger by a factor of $\Gamma^{2}$. Moreover the photons in the local frame are softer by a factor of $\Gamma$. A detailed analysis (Lithwick and Sari 2001) shows that the optical depth can be reduced by effects of relativistic motion by a factor $\sim \Gamma^{6.5}$ where the exact value of the exponent depends on the GRB spectrum. The analysis of Lithwick and Sari yields lower limits on GRB Lorentz factors of several hundreds, the highest Lorentz factors in the universe.

A second hard constraint is the so-called "baryon loading problem". The attainable bulk Lorentz factor is determined by the ratio of available energy and rest mass energy. If an energy of $10^{51} \mathrm{erg}$ is available the fireball cannot contain more than $10^{-5} \mathrm{M}_{\odot}$ in baryonic material, otherwise the required Lorentzfactors will not be reached. This poses a hard problem for central engine models: how can a stellar mass object pump so much energy into a region that is essentially devoid of baryons?

The above reasoning has led to the "fireball" internal-external shocks model. This model is rather independent of the nature of the central engine. The latter one is just required to produce highly relativistic outflow, either in the form of kinetic energy or as Poynting flux. The radiation is produced in (collisionless) shocks. These can either occur due to interaction of the outflow with the circumstellar material ("external shocks") or due to interactions of different por- 
tions of the outflow with different Lorentz-factors, so-called "internal shocks". According to the fireball model the GRB is produced via synchrotron radiation (at least in the most simple model version) in internal shocks. The efficiency of internal shocks depends on the variability within the outflow (Kobayashi et al. 1997). A lot of energy remains as kinetic energy in the ejecta and can later be dissipated via external shocks to produce the much longer lasting multiwavelength afterglow. Such an afterglow had been predicted before its observation by several researchers (Paczynski and Rhoads (1993), Meszarosz and Rees (1993, 1997), Vietri (1997)). The simplest afterglow model with an adiabatic, relativistic blast wave (Blandford and McKee 1976) and synchrotron radiation, seems to fit the bulk of afterglow observations reasonably well (for a detailed discussion see Piran (1999) and Meszaros (2002)).

Detailed investigations of Kobayashi et al. (1997) showed that the GRB lightcurve reflects essentially the activity of the inner engine that produces the relativistic outflow. That means that the engine itself has to produce an erratic sequence of pulses and, whatever the progenitor is, it should be able to produce, depending on its specific system parameters a large variety of different outcomes. This result seems to rule out "one-bang" models, e.g. simple phase transitions in neutron stars.

For an alternative to the fireball model see Dar and De Rujula (2003).

\section{Models for the Central Engine}

Many many GRB models have been suggested over the years and it is impossible to do justice to all of them here. I will therefore just pick out a few that I consider to be particularly interesting.

When discussing the central engine it is worth keeping in mind that the overall burst duration, $\tau$, is -both for long and for short bursts- very long in comparison to the variability time scale, $\delta t$, on which the energy output changes substantially, $\delta t / \tau \ll 1$. This requires the engine to provide (at least) these two different time scales. One (but not the only) reasonable possibility is that the central engine consists of a new-born, stellar mass black hole surrounded by an accretion disk. In this view the dynamical time scale close to the hole sets the variability while the viscous accretion time scale sets the overall burst duration. The hole/disk masses then distinguishes between long and short duration bursts. While this is certainly reasonable, I want to stress here that plausible models do not necessarily have to involve a black hole, examples of such alternative models are Usov's (1992) highly magnetised pulsar formed in an accretion induced collapse or the temporarily stabilised central object resulting in a neutron star coalescence, see below.

One important question is whether there is a single progenitor that produces (depending on say its initial conditions like the rotation rate) either a short or 
a long GRB or whether the two burst classes result form two different progenitor systems. An example of a model that produces both long and short GRBs from one progenitor has been suggested by Yo and Blackman (1998). In their model the GRB is driven by dissipation of Pointing flux extracted from a young, highly magnetised millisecond pulsar formed in an accretion-induced collapse of a white dwarf. If the initial rotation is above a critical rotation rate, the spin-down time scale is determined by gravitational wave emission leading to sub-second spin-down, otherwise its is governed by electromagnetic dipole emission and leads to a much longer duration ( $\gg 1 \mathrm{~s}$ ).

There are, however, reasons to assume that the two GRB classes are caused by different progenitor systems since there are, apart from the duration further substantial differences. First, as mentioned above, short GRBs seem to be systematically harder than their long-duration cousins. Moreover their lightcurves exhibit fewer sub-pulses and they show a different temporal spectral evolution (Norris et al. 2000). Apart from that the spectral break energies of short bursts seem to be larger than for long bursts (Paciesas et al. 2000). As the spectral break energy is sensitive to the cosmological redshift and the Lorentz-factor of the ejecta producing the burst, the higher spectral break energies could mean that either short GRBs have higher Lorentz-factors or/and that their population is closer, i.e. they have, on average, a lower redshift. Both of these possibilities are compatible with compact binary mergers: due to their inspiral time they could occur relatively late in the age of the universe and therefore be at lower redshifts than long GRBs. Moreover, they are not engulfed in a stellar mantle like in the case of a stellar collapse and the high rotation velocities that naturally occur will centrifugally evacuate the region around the binary rotation axis. This fact will make it easier to obtain higher Lorentz-factors without being slowed down by a stellar mantle that has to be penetrated first. Moreover, the two classes seem to be differently distributed in space (Cohen, Kolatt and Piran 1994), consistent with short GRBs being closer than their long-duration cousins. All this hints to two different progenitor systems.

\section{Long-soft Bursts}

Around $70 \%$ of the observed GRBs are the long-soft type. They typically occur at cosmological redshifts of $z \sim 1$ and are believed to be beamed with half-opening angles of $\sim 5^{\circ}$. Thus, we see on average one burst per 300 beamed GRBs with random jet orientations, i.e. the true rate of long GRBs is around 1000 per day in the universe.

Collapsars. The probably most popular GRB-model is the "Collapsar"/"failed supernova" model (Woosley 1993, MacFadyen and Woosley 1999), sometimes also referred to as "hypernova" (Paczynski 1998).

The exact supernova mechanism is despite intense research not known with 


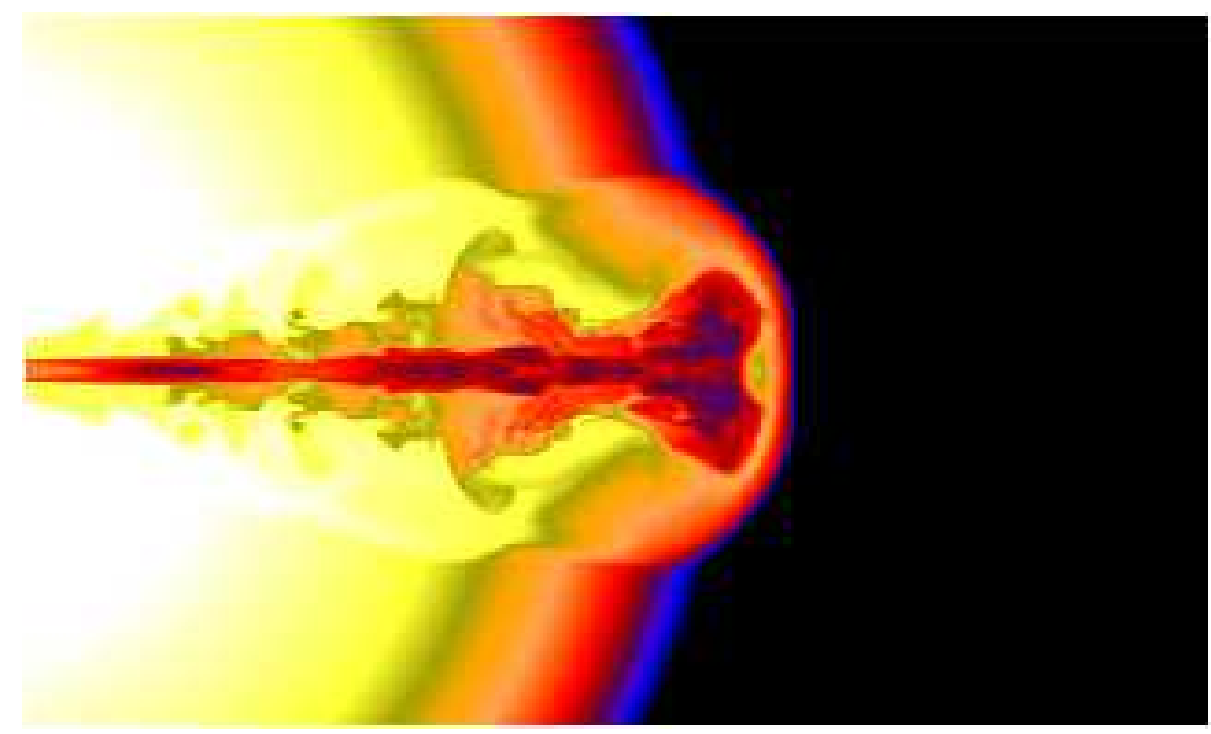

Figure 2. Density distribution of a collapsar jet breaking through the stellar surface. From Zhang, Woosley and Heger 2003.

certainty, but the general believe is that the huge neutrino luminosities from the newborn proto-neutron star will revive the shock that got stalled in the stellar mantle and will drive the explosion. If, however, a black hole rather than a neutron star is formed early on, the neutrino source is essentially shut off and the supernova might "fail". For a non-/slowly rotating star the stellar matter can essentially unhindered disappear in the event horizon of the newborn black hole. If the angular momentum of the star is too high, a disk will form at too large distances to be hot enough for efficient neutrino emission. For intermediate values of angular momentum, however, a more compact, hot accretion disk may form close to the hole. In this case an accretion- rather than neutrino-driven supernova explosion might occur. Although there is no generally accepted mechanism for its explanation there seems to be a common connection between accretion disks and jets. Like in many other astrophysical environments such as active galactic nuclei and young stellar objects the formation of a jet is also plausible in the collapsar case. It might be powered by energy pumped into the polar region above the hole either via the annihilation of neutrinos emitted from the inner, hot parts of the accretion disk or via MHD mechanisms. Initially the density along the original rotation axis is too high for any plausible jet to overcome the ram pressure of the infalling material. But after a few seconds a channel along the rotation axis opens up allowing later on for jet propagation through the star. This channel is expected to collimate 
the escaping jet. If the hole, however, has received too large a kick at birth the energy deposition will not be along the evacuated channel and the jet may be spoilt.

Apart from having the right amount of angular momentum the progenitor star is required to have lost its envelope, since a jet powered for the typical duration of a long burst, $\sim 20 \mathrm{~s}$, could not penetrate a giant star, it would rather dissipate its energy in the stellar envelope. To penetrate say a red supergiant it would take around $1000 \mathrm{~s}$ and it is hard to see why the burst after having been on for so long should then suddenly shut down within $20 \mathrm{~s}$ after breaking through the stellar surface. Therefore, the progenitor stars is expected to have lost its envelope and the GRB is expected to go along with a supernova of type I (i.e. core-collapse but no hydrogen lines). Resulting from very massive stars, collapsars would occur naturally in star forming regions and, as observed, to be associated with a core-collapse supernova of type $\mathrm{Ib} / \mathrm{c}$.

The first simulations of the collapsar scenario have been performed using 2D Newtonian, hydrodynamics (MacFadyen \& Woosley 1999) exploring the collapse of helium cores of more than $10 \mathrm{M}_{\odot}$. In their 2D simulation MacFadyen \& Woosley found the jet to be collimated by the stellar material into opening angles of a few degrees and to transverse the star within $\sim 10 \mathrm{~s}$. The accretion process was estimated to occur for a few tens of seconds. In such a model variability in the lightcurve could result for example from (magneto-) hydrodynamic instabilities in the accretion disk that would translate into a modulation of the neutrino emission/annihilation processes or via Kelvin-Helmholtz instabilities at the interface between the jet and the stellar mantle.

These initial, Newtonian simulations were plagued by highly superluminal motions. Aloy et al. (2000) have improved on this problem using a special relativistic hydrodynamics code to follow jet propagation through a progenitor star. Recently Zhang et al. (2003) have simulated the propagation and breakout of a jet through a Wolf-Rayet star using a 3D, special relativistic grid code. They particularly addressed the question of jet stability going from 2D to 3D and found the gross jet features to be robust against this change. Moreover, they found that even a slowly precessing jet (say a degree per second) is able to penetrate the star and emerge relativistically at its surface. If the jet precesses faster, it is dissipated inside the stellar envelope. At outbreak the jet is surrounded by a mildly relativistic cocoon that will give rise to a transient signal. If the outbreak is seen at a larger angle with the jet axis it may appear as a so-called "X-ray flash" (Heise 2003)

Despite its obvious success there remain several open questions. First and most important is the progenitor question. Do stars with the right amount of angular momentum at collapse exist at all? If state of the art progenitor models in which angular momentum loss through magnetic torques and wind losses are accounted for (Heger and Woosley 2003) are taken at face value, then the 
collapse will not result in a sufficiently massive disk to produce a collapsar. However, as the progenitor uncertainties are substantial that does not necessarily mean a killer argument for collapsars. Another essentially unsolved problem is the question how the jet is launched. In current numerical models the jet formation is not modelled, jets are put in by hand and then their propagation through the star is followed. Further uncertainties come from the numerical resolution. As short-wavelength perturbations grow fastest in a Kelvin-Helmholtz instability the question remains whether current models can already resolve the smallest physically important scales. But this will certainly be addressed in future work. As in many current astrophysical simulations the first collapsar models ignored the influence of magnetic fields. Currently a lot of effort is put into MHD-simulations of this event (see e.g. Proga et al. 2003).

Supranova. Vietri and Stella (1998) have suggested a two stage process to create a GRB ("supranova"). The massive star collapses in a "traditional" supernova to form a rotationally supported neutron star that is beyond its nonrotating upper mass limit. They assume the star to spin down via magnetic dipole radiation. Using a typical neutron star magnetic field of $10^{12} \mathrm{G}$ they estimate a time scale of several years before the "supra-massive" configuration collapses into a black hole. The supernova is expected to leave a $\sim 0.1$ $\mathrm{M}_{\odot}$ accretion disk in orbit around the neutron star and, once the neutron star collapses to form a black hole, the disk might help to extract the rotational energy of the new-born black hole and to launch the GRB.

The strengths of the model are its natural connection to supernovae and star formation and that the supernova remnant would have enough time to form iron via the decay of nickel and cobalt to possibly produce the claimed iron lines. Moreover, it is expected to be a baryon-clean environment. The model is, however, very sensitive to the fine tuning of parameters. Moreover, GRB030329 places a rather strict limit of a few hours on the delay between the $\mathrm{SN}$ and the GRB and thus rules out the supranova model for at least this particular burst.

Fragmenting Core-collapse. Another two stage process, the fragmentation of a core collapse supernova has been suggested recently by Davies et al. (2002). The main idea is that, analogous to the fragmentation of a collapsing molecular cloud into several stars, a sufficiently rapidly rotating progenitor star might undergo fragmentation and form several "lumps" rather than a clean and more or less spherically symmetric neutron star. The various fragments would then be driven towards coalescence via gravitational wave emission and would emit a characteristic chirp signal during inspiral. Once coalesced they will form a "standard GRB central engine", a new-born black hole and an accretion disk. Generally the black hole will receive a kick at birth, a successful GRB-jet can only be launched if the received kick velocity is small. For plau- 
sible system parameters a delay of several hours between supernova and GRB can be obtained. This is well within the current limits set by GRB030329. This model shares with the two previously discussed models its connection with star formation regions and core-collapse supernovae. The exact conditions under which such a fragmentation can occur and the fraction of core-collapse systems that will undergo fragmentation remains to be explored in future work.

\section{Short-hard Bursts}

As outlined above, the class of short-hard GRBs probably results from a different central engine than long bursts. Compact binaries, either two neutron stars [17] or a neutron star and a low mass black hole [47], are the most promising candidates for the progenitor of this class. Their gravitational binding energy of a few times $10^{53}$ erg is expected to easily satisfy the energy requirements of a short GRB (the exact energy in the burst is at present not known since so far no redshift has been detected). As the neutron star dynam-

ical time scale is $\tau_{d y n}=(G \bar{\rho})^{-\frac{1}{2}} \sim 3 \cdot 10^{-4}$ s there should be no problem in providing even the shortest time scales observed in bursts. Moreover, the merger remnants are attractive GRB central engines as the black hole (either preexisting or forming after a neutron star binary coalescence) is guaranteed to be rapidly rotating, either from the angular momentum that has been fed into the hole in the form of neutron star debris or from the orbital motion of a neutron star binary. A soft equation of state leads to more compact neutron stars, therefore a neutron star binary system will have higher orbital velocities at contact and thus a more rapidly rotating black hole will form. The energy stored in the black hole rotation can then possibly be extracted, e.g. via the Blandford-Znajek mechanism (1977).

With the recent discovery of another highly relativistic binary pulsar (Burgay et al. 2003) the estimates for neutron star merger rates have, once again, been increased. With the most likely rates estimated to be as high as $\sim 2 \cdot 10^{-4}$ per year and galaxy (Kalogera et al. 2003), neutron star binary mergers will have no problem to account for all short GRBs, even if substantial beaming is involved.

Neutron star black hole mergers are most often considered to be just a variant of the binary neutron star theme in the sense that they will also produce a black hole plus torus system. It is worth pointing out, however, that the dynamics of this merger process is much more complex than in the neutron star merger case as it is governed by the interplay of gravitational radiation (trying to decrease the orbital separation), mass transfer (trying to increase the orbital separation) and the reaction of the stellar radius to mass loss. The dynamics of this process is very sensitive to the mass ratio of the binary components which is much less constrained than in the binary neutron star case. 
Neutron Star Binary Mergers. Neutron star binaries have for a long time been the model for the central engine of GRBs (Goodman et al. 1987, Eichler et al. 1989, Narayan et al. 1992). With the connection of long GRBs with supernovae being well established by now, compact binary mergers are these days not usually considered to be responsible for long duration GRBs, but they are still the leading model for short GRBs.

The coalescence is an intrinsically three-dimensional phenomenon and therefore analytical guidance is rare (although very welcome) and one has to resort to large scale numerical computations. Additional complications arise from the fact that there is almost no field of astrophysics that does not enter at some stage during the coalescence process: the last stages and the merger are certainly dominated by strong-field general relativistic gravity, the neutron star material follows the laws of hydrodynamics, particle physics enters via possible condensates of "exotic" matter in the high-density interiors of the neutron stars and the copiously produced neutrinos in the hot and dense neutron star debris, questions concerning element formation require detailed information on nuclear structure and reactions (often far from stability) to be included and also magnetic fields might play a decisive role since they may, via transport of angular momentum, determine whether and/or when the central, coalesced object collapses into a black hole. Moreover, they are expected to be amplified in the complex fluid motions following the merger to field strengths where they become dynamically important.

Due to this complexity current investigations follow one of two "orthogonal" lines: either ignoring microphysics, resorting to the simplest equations of state (EOS), polytropes, and thereby focusing on solving the complicated set of general relativistic fluid dynamics equations (or some approximation to it; e.g. Shibata and Uryu 2000, Oechslin et al. 2001) or, along the other line, using accurately treatably (Newtonian) self-gravity of the fluid and investigating the influences of detailed microphysics and relating the merger event to astrophysical phenomena (e.g. Ruffert et al. 2001, Rosswog et al. 2002).

How can the available energy now be transformed into outflowing relativistic plasma after such a coalescence event? A fraction of the energy released as neutrinos is expected to be converted, via collisions, into electron-positron pairs and photons and, if converted in a region of low baryon density, this will give rise to a relativistic wind. Alternatively, strong magnetic fields anchored in the dense matter could convert the available energy of the system into a Poynting-dominated outflow.

Neutrino annihilation

The merger remnant emits neutrinos in copious amounts. The total neutrino luminosities are typically around $2 \cdot 10^{53} \mathrm{ergs} / \mathrm{s}$ with electron-type antineutrinos carrying away the bulk of the energy. Typical neutrino energies 

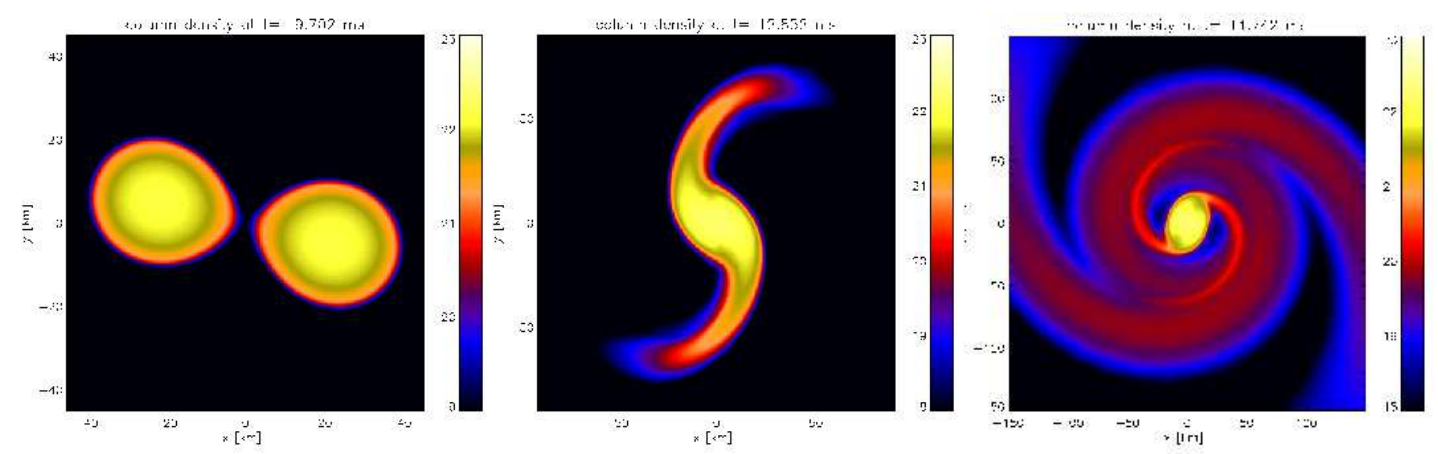

Figure 3. Coalescence of a corotating neutron star binary system $\left(1.4 \mathrm{M}_{\odot}\right.$ each star). Colourcoded is the column density, the axes are in kilometers. The simulations are described in detail in Rosswog et al. 2002.

are not too different from those of type II supernovae, electron neutrinos are around $8 \mathrm{MeV}$, their anti-particles around 15 and the heavy lepton (anti-)neutrinos have energies around $22 \mathrm{MeV}$ (Rosswog and Liebendoerfer 2003).

Neutrino annihilation is an attractive process to launch a fireball as neutrinos can leave the hot remnant easily and can deposit their energy possibly in a region devoid of baryons to avoid prohibitive baryon loading. The centrifugally evacuated funnel region above the remnant is an ideal place for this deposition to occur because it is close to the central energy source (the energy deposition rate scales roughly with the inverse fourth power of the distance) but contains only a small number of baryons (e.g. Ruffert et al. 1997, Rosswog and Davies 2002). Here the $\nu \bar{\nu}$ energy flux can be transformed into a radiation-dominated fluid with a high entropy per baryon. The thick disk geometry of the remnant with its steep density gradients in the radial direction does not allow for lateral expansion. The only escape route is along the initial binary rotation axis. The disk geometry is therefore responsible for channelling the relativistic outflow into a pair of anti-parallel jets. This mechanism is similar to that envisaged by MacFadyen \& Woosley (1999) for the collapsar scenario, but offers the advantage that the jets in the merger case do not have to pierce through a surrounding stellar mantle.

Neutrino annihilation from full merger simulations has been calculated by Ruffert et al. (1997) and Rosswog et al. (2003). Popham et al.(1999) have studied the annihilation for the case of steady state accretion disks. The resulting bulk Lorentz-factor is determined by the annihilation energy deposited per rest mass energy. This quantity is displayed in Figure 4 (for details see Rosswog et al. 2003). The encountered Lorentz-factors range from around 


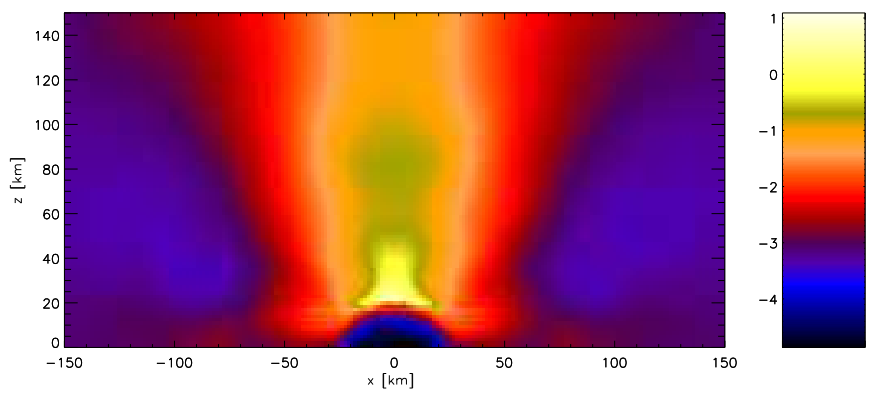

Figure 4. The annihilation of neutrino-antineutrino pairs above the remnant of a neutron star merger drives relativistic jets along the original binary rotation axis (only upper half-plane is shown). The $\mathrm{x}$-axis lies in the original binary orbital plane, the dark oval around the origin is the newly formed, probably unstable, supermassive neutron star formed in the coalescence. Colour-coded is the asymptotic Lorentz-factor. Details can be found in Rosswog et al. 2003.

10 (this is a lower limit as finite numerical resolution leads us to overestimate the funnel density and therefore to underestimate the Lorentz-factor) to a few times $10^{4}$ for extreme cases (see Rosswog et al. 2003 for details), so Lorentz factors of several hundred should not pose any problem for this mechanism.

The total energy in the relativistic outflow, however, is only of order $10^{48} \mathrm{ergs}$ and therefore moderate by GRB standards. Ruffert et al. (1997) find somewhat higher values, but agree that neutrino annihilation can only power a relatively weak burst. To account for the observed luminosities of a short GRB, the relativistic outflow has to remain well-collimated. One such collimation mechanism (Levinson and Eichler 2000) relies on the beaming of the relatively weak, relativistic jet by the ram-pressure of the non-relativistic, but powerful baryonic wind that is blown off the merger remnant via neutrinos (this is very similar to the neutrino-driven wind of a new-born proto-neutron star). Using a theoretical neutron star mass distribution Rosswog and Ramirez-Ruiz (2003) have calculated the resulting beaming angle and the apparent luminosity distribution. They find a broad distribution of apparent luminosities (typical values for isotropized luminosities of $\sim 5 \cdot 10^{50} \mathrm{erg} / \mathrm{s}$ ) with average jet opening angles of $\sim 5^{\circ}$. These results are compatible with both the fluences observed for short GRBs and with the estimated neutron star merger rates. 


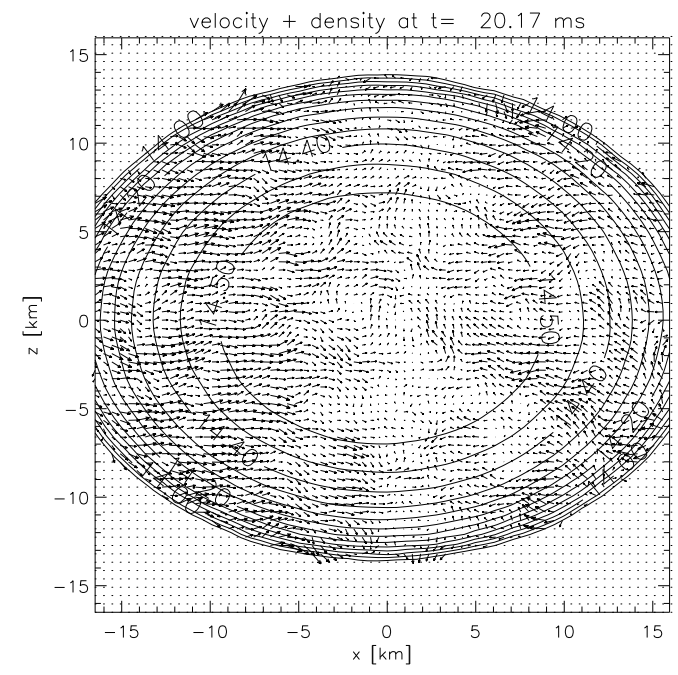

Figure 5. Velocity field (space-fixed frame) inside the central object of the remnant of a neutron star coalescence. The labels at the contour lines refer to $\log (\rho)$, typical fluid velocities are $\sim 10^{8} \mathrm{~cm} / \mathrm{s}$.

\section{Magnetic mechanisms}

Neutron stars are endowed with strong magnetic fields, typically of order $10^{12} \mathrm{G}$. Even the sun with its comparatively moderate magnetic fields and fluid motions produces a large spectrum of different magnetic activities with sometimes violent outbreaks. Thus it is natural to expect scaled up versions such activity in an event as vigorous as a neutron star merger. Several magnetic GRB scenarios have been discussed over the years, e.g. by Usov (1992, 1994), Narayan et al. (1992), Duncan and Thompson (1992), Thompson and Duncan (1994), Meszaroz and Rees (1997), Katz (1997), Kluzniak and Ruderman (1998) and Rosswog et al. (2003).

I want to mention here three different possibilities: first, that the central object formed in the coalescence remains stable for (at least) a short time of the order a second before collapsing to black hole. During this time the seed magnetic fields can be amplified drastically and the central object can, as some kind of a "superpulsar", launch a short lived relativistic wind. Second, similar field amplification processes are expected to occur in the accretion torus around the central object. Finally, if the central object should collapse immediately into a black hole (i.e. before its rotational energy can be extracted by the superpulsar mechanism) its rotational energy can be extracted via a magnetic coupling to the accretion torus. The latter two processes have been discussed in the literature (e.g. Blandford and Znajek 1977, Narayan et al. 1992, Rosswog et al. 
2003). We will therefore draw attention to the first possibility.

The central object of the remnant is rapidly differentially rotating (see Rosswog and Davies 2002 for rotation curves) with rotational periods ranging from $\sim 0.4$ to $\sim 2 \mathrm{~ms}$. Differential rotation is known to be very efficient in stabilising stars that are substantially more massive than their non-rotating maximum mass. For example, Ostriker and Bodenheimer (1968) constructed differentially rotating white dwarfs of $4.1 \mathrm{M}_{\odot}$. A recent investigation analysing differentially rotating polytropic neutron stars (Lyford et al. 2002) finds it possible to stabilise systems even beyond twice the typical neutron star mass of 2.8 $\mathrm{M}_{\odot}$. The exact time scale of this stabilisation is difficult to determine, as all the poorly known high-density nuclear physics could influence the results, but for this process to work, only a time scale of about a second is needed (some authors have estimated time scales of up to years [71]).

It is worth pointing out that the fluid flow in our calculations of the merger (Rosswog et al. 2003) never becomes axisymmetric during the simulation and therefore Cowlings anti-dynamo theorem does not apply here. When the surfaces of the neutron stars come into contact, a vortex sheet forms between them across which the tangential velocities exhibit a discontinuity. This vortex sheet is Kelvin-Helmholtz-unstable. These fluid instabilities lead complex flow patterns inside the central object of the merger remnant. In the orbital plane they manifest themselves as strings of vortex rolls that may merge (see Fig. 8 in Rosswog and Davies 2002). An example of the flow pattern perpendicular to the orbital plane is shown in Fig. 5. This pattern caused by fluid instabilities exhibits "cells" of size $l_{c} \sim 1 \mathrm{~km}$ and velocities of $v_{c} \sim 10^{8} \mathrm{~cm} / \mathrm{s}$. Moreover, we expect neutrino emission to drive convection as in the case of proto-neutron stars. The neutrino optical depth within the remnant drops very steeply from $\sim 10^{4}$ at the centre to the edge of the central object (see Fig. 11 in Rosswog and Liebendoerfer 2003). For this reason the outer layers loose neutrino energy, entropy and lepton number at a much higher rate than the interior. This leads to a gradual build-up of a negative entropy and lepton number gradient which will drive vigorous convection (e.g. Epstein 1979). We expect this to set in after a substantial fraction of the neutrino cooling time (i.e. on time scales longer than our simulated time) when a lot of the thermal energy of the remnant has been radiated away. With neutrinos as dominant viscosity source we find viscous damping time scales of several tens of seconds, i.e. much longer than the processes we are interested in (see below).

We expect an efficient $\alpha-\Omega$-dynamo to be at work in the merger remnant. The differential rotation will wind up initial poloidal into a strong toroidal field (" $\Omega$-effect"), the fluid instabilities/convection will transform toroidal fields into poloidal ones and vice versa (" $\alpha$-effect"). Usually, the Rossby number, $R o \equiv \frac{\tau_{\text {rot }}}{\tau_{\text {conv }}}$ is adopted as a measure of the efficiency of dynamo action in a star. In the central object we find Rossby numbers well below unity, $\sim 0.4$, 
and therefore expect an efficient amplification of initial seed magnetic fields. A convective dynamo amplifies initial fields exponentially with an e-folding time given approximately by the convective overturn time, $\tau_{c} \approx 3 \mathrm{~ms}$; the saturation field strength is thereby independent of the initial seed field (Nordlund et al. 1992).

Adopting the kinematic dynamo approximation we find that, if we start with a typical neutron star magnetic field, $B_{0}=10^{12} \mathrm{G}$, as seed, equipartition field strength in the central object will be reached (provided enough kinetic energy is available, see below) in only $\approx 40 \mathrm{~ms}$. The equipartition field strengths in the remnant are a few times $10^{17} \mathrm{G}$ for the central object and around $\sim 10^{15}$ $\mathrm{G}$ for the surrounding torus (see Fig. 8 in Rosswog et al. 2003). To estimate the maximum obtainable magnetic field strength (averaged over the central object) we assume that all of the available kinetic energy can be transformed into magnetic field energy. Using the kinetic energy stored in the rotation of the central object, $E_{\text {kin }}=8 \cdot 10^{52}$ erg for our generic simulation, we find $\left\langle B_{c o}\right\rangle=\left(3 \cdot E_{\mathrm{kin}} / R_{c o}^{3}\right)^{1 / 2} \approx 3 \cdot 10^{17} \mathrm{G}$, where $R_{c o}$ is the radius of the central object (note that if only a fraction of 0.1 of the equipartition pressure should be reached this would still correspond to $\sim 10^{17} \mathrm{G}$ ).

There are various ways how this huge field strength could be used to produce a GRB. The fields in the vortex rolls (see Fig. 8 in Rosswog and Davies 2002) will wind up the magnetic field fastest. Once the field reaches a strength close to the local equipartition value it will become buoyant, float up, break through the surface and possibly reconnect in an ultra-relativistic blast (Kluzniak and Ruderman 1998). The time structure imprinted on the sequence of such blasts would then reflect the activity of the fluid instabilities inside the central object. The expected lightcurve of the GRB would therefore be an erratic sequence of sub-bursts with variations on millisecond time scales.

Simultaneously such an object can act as a scaled-up "super-pulsar" and drive out an ultra-relativistic wind. A similar configuration, a millisecond pulsar with a magnetic field of a few times $10^{15} \mathrm{G}$, formed for example in an accretion-induced collapse, has been suggested as a GRB-model by Usov (1992, 1994). The kinetic energy from the braking of the central object is mainly transformed into magnetic field energy that is frozen in the outflowing plasma. At some stage the plasma becomes transparent to its own photons producing a blackbody component. Further out from the remnant the MHDapproximation breaks down and intense electromagnetic waves of the rotation frequency of the central engine are produced. These will transfer their energy partly into accelerating outflowing particles to Lorentz-factors in excess of $10^{6}$ that can produce an afterglow via interaction with the external medium. The other part goes into non-thermal synchro-Compton radiation with typical energies of $\sim 1 \mathrm{MeV}$ (Usov 1994). 

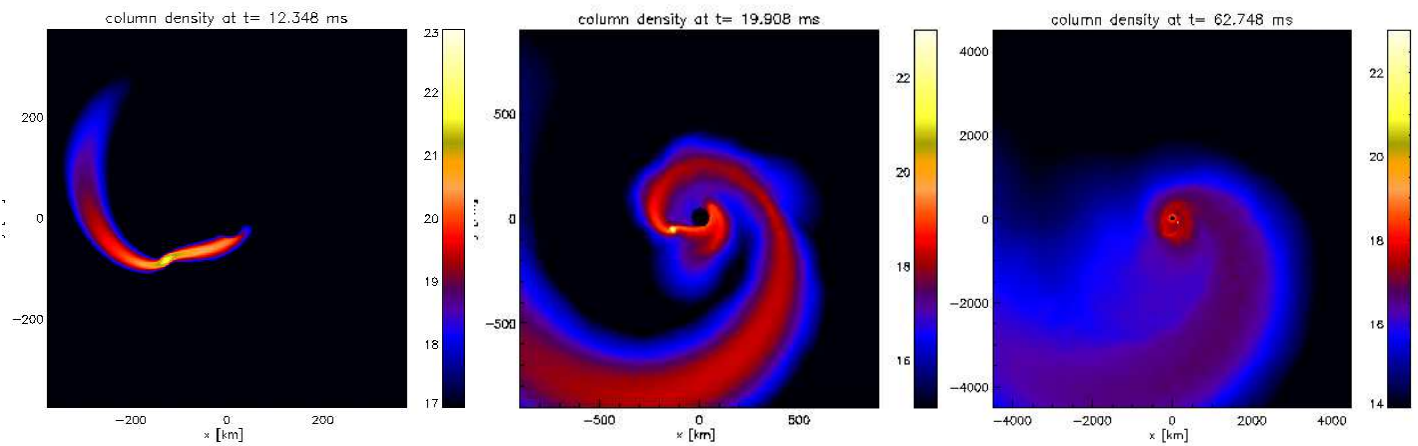

Figure 6. Tidal disruption of $1.4 \mathrm{M}_{\odot}$ neutron star by a $14 \mathrm{M}_{\odot}$ black hole. Colour-coded is the logarithm of the column density $\left(\mathrm{g} / \mathrm{cm}^{2}\right)$. Note the change in the scales $(\mathrm{km})$ of the different panels. From Rosswog et al. 2004.

Neutron Star Black Hole Mergers. Neutron star black hole mergers have been estimated to occur at a rate of $10^{-4}$ (year galaxy) ${ }^{-1}$ [2], i.e. an event rate comparable to that of neutron star binaries is expected. Moreover, their gravitational wave signal is stronger and therefore they could possibly dominate the first detectable signals from inspirals of compact binaries.

Simulations of this scenario have been performed by Lee [30-32] using Newtonian gravity and polytropic equations of state of varying stiffness. Ruffert, Janka and Eberl performed similar simulations but with a detailed microphysics input (nuclear equation of state and neutrino leakage [25]). In our own simulations of NS-BH mergers we used a relativistic mean field equation of state [70] together with three-dimensional smoothed particle hydrodynamics and a detailed, multiflavour neutrino treatment.

The interaction of mass transfer, gravitational wave backreaction and the reaction of the neutron star radius to the mass loss leads to a very complicated accretion dynamics in a neutron star black hole system. We find in all of our simulations (apart from an extreme test case with mass ratio $q=0.93$, i.e. a black hole of $1.5 \mathrm{M}_{\odot}$ and a neutron star of $1.4 \mathrm{M}_{\odot}$ ) that a "mini-neutron star" survives. A specific example, a black hole with $14 \mathrm{M}_{\odot}$, an initially tidally locked neutron star of $1.4 \mathrm{M}_{\odot}$ modelled using Newtonian gravity plus gravitational wave backreaction forces is shown in Figure 6. We find initial peak mass transfer rates of $\sim 500 \mathrm{M}_{\odot} / \mathrm{s}$ (at about the time shown in the first panel of Figure 6). After that phase the orbit widens, and we find a long sequence of mass transfer episodes, always transferring mass into an accretion disk around the hole, moving out, spiralling in again and so on. At the end of the simulation (after around $64 \mathrm{~ms}$ and seven mass transfer phases) the neutron star 
still has around $0.2 \mathrm{M}_{\odot}$ and shows no sign of being disrupted soon (the later mass transfer rates reduce the neutron star mass only by small amounts). The accretion disk that forms around the hole is very low in mass $\left(>10^{-2} \mathrm{M}_{\odot}\right)$ and relatively moderate in temperature $(T \sim 2 \mathrm{MeV})$. Under these circumstances the neutrino luminosity is around an order of magnitude smaller than in the binary neutron star merger case and therefore not very encouraging to

power a GRB. Systems without initial neutron star spins yield somewhat more promising accretion disks, but details remain to be explored further. These results are sensitive to the stiffness of the equation of state, a softening of the EOS at higher densities (e.g. due to the presence of hyperons) could possibly substantially alter the results.

\section{Summary and Prospects}

In the last few years the GRB field has seen tremendous progress. The cosmological distance scale is now well-established. Moreover, since GRB030329 the connection of (long) GRBs with supernovae, suspected already in the very first paper on GRBs by Klebesadel et al. (1973), has been put to a firm footing. This lends further strength to the collapsar model of the long burst category. But there are still myriards of open questions both related to the astrophysical events/central engines as well as related to the fundamental physics involved. For example, is our picture of stellar evolution wrong if state of the art progenitor models cannot produce progenitors of sufficient angular momentum for a collapsar? If collapsars result from Wolf-Rayet stars then why does the expected ambient matter density distribution resulting from a stellar wind $\left(\rho \propto r^{-2}\right.$ ) not give better results in interpreting observations than a constant ambient matter density? What is the connection between GRBs and X-ray flashes? Is it the same event seen from different angles? Do short GRBs have afterglows? Why are their spectra harder? Do they also occur close to star froming regions?

Or from the physics side: by which physical mechanisms are jets launched from accretion disks? How are the ejecta accelerated to Lorentz factors beyond 100? How are the magnetic fields at the emission site created? Are they advected from the central engine or are they produced by in shocks?

Hopefully, at least some of the above questions can be answered in the near future by missions such as SWIFT.

\section{Acknowledgements}

The simulations of the compact binary mergers were performed using both the UK Astrophysical Fluids Facility (UKAFF) and the University of Leicester Mathematical Modelling Centre's supercomputer. S.R. gratefully acknowledges a PPARC Advanced Fellowship. 


\section{References}

[1] Band, D. et al., ApJ, 413, 281 (1993)

[2] Bethe, H.A. and Brown, G.E., ApJ, 506, 780 (1998)

[3] Blandford, R. and McKee,C.F. Physics of Fluids, 19, 1130 (1976)

[4] Blandford, R. and Znajek, MNRAS, 179, 433 (1977)

[5] Bloom, J.S. et al., Nature, 401, 453 (1999)

[6] Bloom, J.S., Kulkarni, S.R. and Djorgovski, S.G., AJ, 123, 1111 (2002)

[7] Burgay, M. et al., Nature, 426, 531 (2003)

[8] Coburn, W. and Boggs, S.E., Nature, 423, 415 (2003)

[9] Cohen, E., Kolatt,T., Piran, T.,astro-ph/9406012 (1994)

[10] Cohen, E. et al., ApJ, 480, 330 (1997)

[11] Costa, E., Nature, 372, 652 (1997)

[12] Davies, M.B., King, A.R., Rosswog, S. and Wynn, G., ApJ, 579, 63 (2002)

[13] Dar, A. and De Rujula, A., astro-ph/0308248 (2003)

[14] Duncan, R.C. \& Thompson, C., ApJ, 392, L9 (1992)

[15] Epstein, R.I., MNRAS, 188, 305 (1979)

[16] Ghisellini et al. A\&A, 389, L33 (2002)

[17] Eichler,D. et al., Nature, 340, 126 (1989)

[18] D. Frail et al., ApJ, 526, 152 (1999)

[19] Fryer, C.L., Woosely, S.E. and Hartmann, D.H., ApJ, 526, 152 (1999)

[20] Goodman, J., ApJ, 308, L46 (1986)

[21] Goodman, J., Dar, A. and Nussinov, S., ApJ, 314, L7 (1987)

[22] Heger, A. and Woosley, S.E., GRBs in the Afterglow Era, ESO Astrophysics Symposia, AIP Conference Proceedings, Volume 662, p. 214 (2003)

[23] Heise, J., GRBs in the Afterglow Era, ESO Astrophysics Symposia, AIP Conference Proceedings, Volume 662, p. 229 (2003)

[24] Hjorth, J. et al, Nature, 423, 847 (2003)

[25] Janka, H.T. et al., ApJ, 527, 39 (1999)

[26] Kalogera, V. et al., submitted to ApJL, astro-ph/0312101 (2003)

[27] Klebesadel, R.W., Strong, I.B. and Olson, R.A., ApJ, 182, L85 (1973)

[28] Kobayashi, S., Piran, T. and Sari, R., ApJ, 490, 92 (1997)

[29] Kouveliotou, C., et al., ApJ, 413, L101 (1993)

[30] Lee, W.H. and Kluzniak, W.L., ApJ, 526, 178 (1999)

[31] Lee, W.H. and Kluzniak, W.L., MNRAS, 308, 780 (1999)

[32] Lee, W.H., MNRAS, 328, 583 (2001)

[33] Lloyd-Ronning, N.M., Fryer, C.L. and Ramirez-Ruiz, E., ApJ, 574, 554 (2002)

[34] MacFadyen, A.I. and Woosley, S.E., ApJ, 524, 262 (1999)

[35] MacFadyen, A.I., Woosley, S.E. and Heger, A., ApJ, 550, 410 (2001)

[36] Mao, S., Narayan, R. and Piran, T., ApJ, 420, 171, (1994)

[37] Meszaros, P. \& Rees, M. J., ApJ, 418, L5 (1993) 
[38] Meszaros, P. \& Rees, M. J., ApJ, 476, 232 (1997)

[39] Meszaros, P. \& Rees, M. J., ApJ, 482, L29 (1997)

[40] Meszaros, P., Ann. Rev. of A \& A, 40, 137 (2002)

[41] Narayan, R., Pazcynski, B. and Piran, T., ApJ, 395, L83 (1992)

[42] Norris, J.P., Marani, G.F. and Bonnell, J.T., ApJ, 534, 248 (2000)

[43] Oechslin, R., Rosswog, S. and Thielemann, F.-K., Phys. Rev. D, 65, 103005 (2002)

[44] Paciesas, W.S. et al., ApJS, 122, 465 (1999)

[45] Paciesas, W.S. et al., Gamma-ray Bursts in the Afterglow Era, Proceedings of the International GRB conference in Rome; Berlin Heidelberg, Springer, 2001

[46] Paczynski, B., ApJ, 308, L43 (1986)

[47] Paczynski, B., Acta Astronomica, 41, 257, (1992)

[48] Paczynski, B. and Rhoads, J.E., ApJ, 418, L5 (1993)

[49] Piran, T., Phys. Rep. 314, 575 (1999)

[50] Piro, L., Science, 290, 955 (2000)

[51] Popham, R., Woosley, S.E., Fryer, C., 518,356 (1999)

[52] Preece, R.D. et al., ApJ, 506, 23 (1998)

[53] Proga, D. et al., ApJ, 599, L5 (2003)

[54] Rosswog, S., Liebendoerfer, M., Thielemann, F.-K., Davies, M. B., Benz, W. \& Piran, T., A\&A, 341, 499 (1999)

[55] Rosswog S., Davies M. B., Thielemann F.-K., Piran T., A \& A, 360, 171 (2000)

[56] Rosswog S., Davies M. B., MNRAS, 334, 481 (2002)

[57] Rosswog S., Ramirez-Ruiz E., MNRAS, 336, L7 (2002)

[58] Rosswog, S. \& Liebendoerfer, MNRAS, 342, 673 (2003)

[59] Rosswog, S. \& Ramirez-Ruiz E., MNRAS, 343, L36 (2003)

[60] Rosswog, S., Ramirez-Ruiz E. \& Davies, M.B., MNRAS, 345, 1077 (2003)

[61] Rosswog, S. et al., in preparation (2004)

[62] Ruffert M., Janka H.-T., Schafer G., A \& A, 311, 532 (1996)

[63] Ruffert M., Janka H.-T., Takahashi K., Schafer G., A \& A, 319, 122 (1997)

[64] Ruffert M., Janka H.-T., A \& A, 344, 573 (1999)

[65] Ruffert M., Janka H.-T., A \& A, 380, 544 (2001)

[66] Ruderman, M, Ann. N.Y. Acad. Sci., 262, 164 (1975)

[67] Rutledge, R.E. and Fox, D.B., astro-ph/0310385

[68] Schaefer, B.E. and Walker, K.C., ApJ, 511, L89 (1999)

[69] Schmidt, W.K.H., Nature, 271, 525 (1978)

[70] Shen H., Toki H., Oyamatsu K., Sumiyoshi K., Nuclear Physics A, 637, 435 (1998)

[71] Shibata, M. Phys. Rev. D, 60, 104052 (1999)

[72] Shibata, M. and Uryu, K., Phys. Rev. D, 61, 064001 (2000)

[73] Stanek, K.Z. et al., ApJ, 591, L17 (2003)

[74] Thompson, C. \& Duncan, R.C., ApJ, 408, 194 (1993)

[75] Usov, V.V., Nature, 357, 472 (1992)

[76] Vietri, M., ApJ, 478, L9 (1997)

[77] Vietri, M., and Stella, L., ApJ, 507, L45 (1998)

[78] Woosely, S.E., ApJ, 405, 273 (1993)

[79] Yi, I. and Blackman, E.G., ApJ, 494, L163 (1998)

[80] Zhang, W., Woosley, S.E. \& MacFadyen, ApJ, 586, 356 (2003) 